\title{
ATENDIMENTO INFANTOJUVENIL EM CENTROS DE ATENÇÃO PSICOSSOCIAL DE SALVADOR, BAHIA, BRASIL
}

Grey Ceballos ${ }^{a}$

Darci Neves dos Santos ${ }^{b}$

Eduardo Luiz Andrade Mota ${ }^{c}$

\section{Resumo}

Centros de Atenção Psicossocial Infantojuvenis (CAPSi) são equipamentos especializados para dar cobertura à demanda de crianças e adolescentes que requerem algum tipo de tratamento para transtornos mentais. O objetivo do estudo foi descrever o perfil nosológico e sociodemográfico dos registros de atendimentos infantojuvenis realizados pelo conjunto de Centros de Atenção Psicossocial implantados em Salvador, Bahia, Brasil. Tratou-se de estudo ecológico, descritivo. Utilizaram-se dados secundários de 2010 a 2012, de população entre 1 e 19 anos de idade assistida nos Centros de Atenção Psicossocial. Dados das Autorizações de Pagamento de Serviços de Alta Complexidade foram obtidos no site do Departamento de informação do SUS e foram analisados com os aplicativos TabWin e Epilnfo versão 7.0. Realizaram-se análises descritivas gerais. Os resultados informaram que o total de 14 CAPS com registros em Salvador registraram 5.140 atendimentos no período estudado, sendo dois centros para infância e adolescência (CAPSi), 1 para usuários de álcool e outras drogas (CAPS-AD) e 11 equipamentos para adultos. Nos CAPSis, $27,4 \%$ dos atendimentos registrados foram para transtornos do comportamento, $26,8 \%$ para retardo mental e 19,3\% para transtornos do desenvolvimento. Nos registros de CAPS para adultos, as esquizofrenias foram os diagnósticos mais frequentes entre os adolescentes (48,3\%). No CAPS-AD, 80,4\% dos diagnósticos em adolescente correspondeu a transtornos mentais pelo uso de múltiplas substâncias psicoativas. Concluiu-se que o perfil nosológico dos CAPS infantis caracterizou-se pelo predomínio dos transtornos de

a Enfermeira. Mestre em Saúde Coletiva. Docente da Faculdade de Enfermagem. Universidade de Antioquia, Colômbia. E-mail: grey.ceballos@udea.edu.co

b Médica Psiquiatra. Sanitarista. Doutora em Epidemiologia Psiquiátrica. Professora Adjunta do Instituto de Saúde Coletiva. Universidade Federal da Bahia. Salvador, Bahia, Brasil.

c Médico. Mestre em Saúde Pública. Doutor em Medicina. Professor Associado IV do Instituto de Saúde Coletiva. Universidade Federal da Bahia. Salvador, Bahia,Brasil.E-mail: emota@ufba.br

Endereço para correspondência: Rua Catarina Paraguaçu, número 86, Graça, Salvador, Bahia, Brasil. CEP: 40150-200. E-mail: darci@ufba.br 
Revista Baiana de Saúde Pública aprendizagem. O atendimento infantojuvenil em CAPS para adultos sugere déficit do número de CAPSi.

Palavras-chave: Epidemiologia. Serviços comunitários de saúde mental. Crianças e adolescentes.
IN SALVADOR, BAHIA

\begin{abstract}
Children's Psychosocial Care Centers (CAPSi) are specialized equipment to meet the demand of children and adolescents who require some type of treatment for mental disorders. The objective of the study was to describe the nosological and sociodemographic profile of the records from child and adolescent care performed by the set of Psychosocial Care Centers located in Salvador, Bahia, Brazil. This was an ecological, descriptive study. Secondary data were used from 2010 to 2012, from population aged between 1 and 19 years assisted by the Psychosocial Care Centers. Data from Payment Authorizations of High Complexity Services were obtained from the SUS Information Department website and were analyzed with the applications TabWin and Epilnfo version 7.0. General descriptive analysis were carried out. The results indicate that the total of 14 CAPS with records in Salvador registered 5,140 visits in the period studied, two centers for childhood and adolescence (CAPSi), 1 for alcohol and other drugs users (CAPS-AD) and 11 adult equipments. In CAPSis, $27.4 \%$ of the registered visits were for behavioral disorders, $26.8 \%$ for mental retardation and $19.3 \%$ for developmental disorders. In the CAPS records for adults, schizophrenias were the most frequent diagnoses among adolescents (48.3\%). In CAPS-AD, 80.4\% of diagnoses in adolescents corresponded to mental disorders due to the use of multiple psychoactive substances. It was concluded that the nosological profile of childhood CAPS was characterized by the predominance of learning disorders. Child and adolescent care in CAPS for adults suggests scarcity of CAPSi amount.
\end{abstract} Keywords: Epidemiology. Community mental health services. Child and adolescent.

\section{ATENCIÓN INFANTOJUVENIL EN CENTROS DE ATENCIÓN PSICOSOCIAL \\ DE SALVADOR- BAHÍA, BRASIL}

\section{Resumen}

Centros de Atención Psicosocial Infantojuvenil (CAPSi) son dispositivos especializados para dar cobertura a la demanda de niños y adolescentes que requieren algún tipo 
de tratamiento para trastornos mentales. El objetivo del estudio fue describir el perfil nosológico y sociodemográfico de los registros de atenciones infantojuveniles realizadas por el conjunto de Centros de Atención Psicosocial instalados en Salvador, Bahía, Brasil. Se trató de un estudio ecológico, descriptivo. Se utilizaron datos secundarios de 2010 a 2012, de población entre 1 y 19 años atendida en los Centros de Atención Psicosocial. Datos de las Autorizaciones de Pago de Servicios de Alta Complejidad fueron obtenidos en la página del Departamento de información del SUS y fueron analizados con los aplicativos TabWin y Epilnfo versión 7.0. Se realizaron análisis descriptivos generales. Los resultados muestran un total de 14 CAPS con registros en Salvador, estos registraron 5.140 atenciones en el periodo estudiado, siendo dos centros para infancia y adolescencia (CAPSi), 1 para usuarios de alcohol y otras drogas (CAPS-AD) y 11 centros para adultos. En los CAPSis, el 27,4\% de las atenciones registradas era para trastornos del comportamiento, el 26,8\% para retardo mental y el 19,3\% para trastornos del desarrollo. En los registros de CAPS para adultos, las esquizofrenias fueron los diagnósticos más frecuentes entre adolescentes (48,3\%). En CAPS-AD, 80,4\% de los diagnósticos en adolescente correspondían a trastornos mentales por el uso de múltiples substancias psicoactivas. Se concluye que el perfil nosológico de los CAPS infantiles se caracterizó por el predominio de trastornos de aprendizaje. La atención infantojuvenil en CAPS para adultos sugiere déficit do número de CAPSi.

Palabras clave: Epidemiologia. Servicios comunitarios de salud mental. Niños y adolescentes.

\section{INTRODUÇÃO}

Saúde mental de crianças e adolescentes é considerada uma prioridade na agenda de saúde global. Os problemas nessa área são prevalentes em todos os países, contribuindo significativamente para a carga das doenças e a perda de qualidade de vida, com enormes custos econômicos e sociais ${ }^{1}$. Estimativas mundiais sugerem que $20 \%$ de crianças e adolescentes apresentam enfermidades mentais, sendo reconhecida uma variação entre $4 \%$ e $6 \%$ que demandaria intervenção clínica ${ }^{2}$. No Brasil, a prevalência dessas condições varia entre 7,0\% e $24,6 \%^{1,3-5}$. Todavia, constata-se pouco conhecimento sobre a utilização de serviços de saúde mental especializados para esse grupo populacional ${ }^{6-7}$, e isso compromete decisões sobre políticas e programas que assegurem a oferta de cuidados e acesso dos indivíduos afetados e grupos de risco, observando-se disparidades entre necessidades e recursos disponíveis ${ }^{7}$

Estudo realizado em São Paulo estimou que 7,3\% de crianças e adolescentes requeriam algum tipo de tratamento para transtornos mentais, porém a capacidade intersetorial instalada só permitia o atendimento de 14\% da demanda, tornando-se necessário 
Revista Baiana de Saúde Pública aproximadamente 7 anos para atender à necessidade estimada ${ }^{5}$. Outro estudo realizado em 142 municípios brasileiros observou que, dentre as solicitações de atendimento em saúde mental para crianças e adolescentes, 24,4\% não foram realizadas ${ }^{8}$. Sabe-se também que em torno de $67 \%$ dos recursos financeiros mundiais designados para saúde mental são ainda utilizados para internamentos psiquiátricos, apesar da associação deste procedimento com violações dos direitos humanos e baixa efetividade ${ }^{2}$. Nos países em desenvolvimento, os poucos serviços disponíveis são predominantemente baseados em atenção hospitalar e outras instituições tutelares, sendo escassas as redes comunitárias de serviços e apoio² .

Evidências robustas têm demonstrado benefícios da atenção à saúde mental de crianças e adolescentes em ambientes menos restritivos e próximos de suas comunidades ${ }^{9}$. Entretanto, a oferta desses serviços tem sido particularmente lenta na maioria dos países de renda baixa e média, onde $76 \%$ das verbas ainda são destinadas para os hospitais psiquiátricos ${ }^{9}$. No Brasil, a Reforma Psiquiátrica visa substituir os hospitais psiquiátricos por equipamentos comunitários especializados, denominados Centros de Atenção Psicossocial (CAPS). Mediante equipamentos especializados para a infância e a adolescência - Centros de Atenção Psicossocial Infantojuvenis (CAPSi) -, pretende-se dar cobertura à demanda dessa população ${ }^{10}$. Desde o ano 2001, esses centros vêm sendo implantados em todo o país, entretanto seus resultados ainda carecem de avaliação.

Existem, em Salvador, apenas dois CAPSi, o que sugere a realização de atendimentos dessa população em CAPS gerais e especializados para Álcool e outras Drogas. Somente um estudo realizado em 2003 apresenta dados ambulatoriais e de internações hospitalares em Salvador relativos à assistência em saúde mental desse grupo populacional ${ }^{11}$. Pela importância de avaliar aspectos da implementação da reforma psiquiátrica e pela restrição de recursos para atenção comunitária infantojuvenil, considera-se relevante identificar o conjunto de atendimentos infantojuvenis realizados pelos diferentes tipos de CAPS no município. Este conhecimento poderá contribuir para o planejamento e tomada de decisões em relação à organização local dos serviços de saúde mental.

O objetivo deste estudo é descrever o perfil nosológico e sociodemográfico dos registros de atendimentos infantojuvenis realizados pelo conjunto de CAPS implantados em Salvador, Bahia, Brasil.

\section{MÉTODO}

Estudo descritivo, ecológico, realizado com dados secundários das Autorizações de Procedimentos de Alta complexidade (APAC) do Departamento de Informação do Sistema 
Único de Saúde (Datasus) do Ministério da Saúde, no período de janeiro 2010 a dezembro de 2012. Trata-se de dados de sobre atendimentos, em que não é possível a identificação nominal. Os critérios de inclusão das unidades de análise foram: estar classificado como CAPS, independente de sua especialidade; estar localizado no município de Salvador; ter registro de atendimentos de usuários entre 1 e 19 anos de idade nas APAC, no período definido pelo estudo. Não foram incluídos CAPS sem registros de APAC de atendimentos de usuários com idade entre 1 e 19 anos. Participaram 14 CAPS localizados no município, sendo 2 para infância e adolescência (CAPSi), e 1 CAPS-AD especializado no atendimento de usuários dependentes de álcool e outras drogas. Os CAPSis estão localizados nos distritos Sanitários da Liberdade (CAPSi Liberdade) e de Barra/Rio Vermelho (CAPSI Dr. Luiz Meira Lessa), enquanto os demais CAPS de adultos e CAPS-AD, estão distribuídos nos 12 Distritos Sanitários de Salvador.

Para a coleta dos dados, foram acessados os arquivos do Sistema de Informações Ambulatoriais/APAC com dados de domínio público, dissemináveis para tabulação, a partir de janeiro de 2008, nos quais estão disponíveis arquivos com nome AD-APAC de Laudos Diversos, referentes aos anos 2010, 2011, 2012. Os dados foram visualizados mediante o uso do aplicativo TabWin do Datasus. Todas as bases de dados utilizadas para as análises, bem como o TabWin, estão disponíveis no portal Datasus. Os bancos de dados foram processados pelo pacote estatístico Epi Info versão 7.0 (Centers for Disease Control and Prevention, Atlanta, Estados Unidos). Realizaram-se análises descritivas, apresentando-se frequências absolutas e relativas para cada serviço estudado. A pesquisa foi aprovada pelo comitê de Ética do Instituto de Saúde Coletiva da Universidade Federal da Bahia em 2013, sob Parecer n. 182.169.

Foram definidos quatro conjuntos de variáveis:

a) características sociodemográficos: sexo ( $M=$ Masculino, $F=$ Feminino), raça/cor da pele (Branca, Preta, Parda, Amarela); idade (em anos). Constituíram-se 5 grupos etários: 1 a $4 ; 5$ a $8 ; 9$ a 12; 13 a 15 e 16 a 19. Considerou-se infância até 10 anos incompletos e adolescência entre 10 e 19 anos $^{12}$;

b) o atendimento realizado foi classificado conforme dispõe a lei, sendo primeiro atendimento ou consultas subsequentes; além de três modalidades para sua caracterização: intensivo, quando ocorre diariamente, para aqueles com grave sofrimento psíquico; semi-intensivo, quando o usuário comparece até 12 dias por mês; e não intensivo, quando a pessoa não precisa de suporte contínuo, recebendo até três atendimentos mensais ${ }^{13}$;

c) o perfil nosológico foi determinado pelos diagnósticos psiquiátricos que aparecem registrados no banco de dados, utilizando a Classificação Estatística Internacional de 
Revista Baiana de Saúde Pública
Doenças e Problemas Relacionados à Saúde, 10ª Revisão (CID-10), especificamente o grupo V (F00-F99); e

d) cobertura de CAPS: utilizou-se o indicador de cobertura de CAPS preconizado pelo Ministério da Saúde (CAPS/100.000 habitantes) ponderado por tipo de CAPS $^{14}$.

\section{RESULTADOS}

Os resultados do estudo realizado estarão expostos em cinco subseções que tratam das características dos usuários e do atendimento oferecido, do perfil nosológico dos atendimentos em CAPSI, do perfil nosológico do atendimento infantojuvenil em CAPS de adultos e em CAPS-AD e da cobertura de CAPS em Salvador.

\section{CARACTERÍSTICAS DOS USUÁRIOS E DO ATENDIMENTO OFERECIDO}

Registraram-se no período estudado 5.140 atendimentos. Os períodos de registro dos atendimentos no Datasus foram diferentes entre as unidades. No CAPSi Liberdade, havia apenas registros de janeiro a setembro de 2011. Já o CAPSi Dr Luiz Meira Lessa registrou informações de fevereiro de 2010 a fevereiro de 2012. Para os CAPS gerais de adultos e CAPS-AD, encontrou-se informação do período de janeiro de 2010 a dezembro de 2012, sendo irregular a periodicidade do registro de dados.

Os dois serviços especializados para crianças e adolescentes (CAPSi) da cidade realizaram 4.040 atendimentos entre 2010 e 2012. Observou-se uma distribuição similar entre os grupos de idade, com uma mediana de idade de 12 anos, havendo, no CAPSi Liberdade, a tendência de aumento do número de atendimentos com a idade dos usuários. Nos CAPS gerais de adultos e no CAPS-AD, o atendimento de jovens concentrou-se na faixa de 16 a 19 anos, com uma mediana de 18 anos. Na distribuição por sexo, a população masculina foi superior a 50,0\% em todos os CAPS, sendo maior no CAPSi liberdade e CAPS-AD, com 71,7\% e $94,5 \%$, respectivamente. A variável raça/cor da pele teve registro incompleto na maioria dos CAPS, sendo o CAPSi Liberdade o único com registros consistentes. Em todos os CAPS, o tipo mais frequente de atendimento foi o seguimento contínuo, superando 60,0\% dos registros; a modalidade de atendimento mais frequente foi a semi-intensiva, com percentuais acima de 40,0\%, exceto no CAPSi Liberdade, onde foi maior a modalidade não-intensiva (Tabela 1). 
Tabela 1 - Frequência e percentual dos registros de atendimentos nos CAPS* para a população infantojuvenil segundo aspectos sociodemográficos e modalidades de atendimento. Salvador, Bahia, Brasil - 2010-2012

\begin{tabular}{|c|c|c|c|c|c|c|c|c|}
\hline \multirow[t]{2}{*}{ Variável } & \multicolumn{2}{|c|}{ CAPSI Liberdade } & \multicolumn{2}{|c|}{$\begin{array}{c}\text { CAPSI Luiz Meira } \\
\text { Lessa }\end{array}$} & \multicolumn{2}{|c|}{$\begin{array}{l}\text { Outros CAPS } \\
\text { Adultos }\end{array}$} & \multicolumn{2}{|c|}{$\begin{array}{c}\text { CAPS-AD } \\
\text { Pernambués }\end{array}$} \\
\hline & $\mathbf{n}$ & $\%$ & $\mathbf{n}$ & $\%$ & $\mathbf{n}$ & $\%$ & $\mathbf{n}$ & $\%$ \\
\hline \multicolumn{9}{|l|}{ Faixa etária (anos) } \\
\hline $1-4$ & 69 & 5,7 & 121 & 4,3 & 29 & 3,1 & - & - \\
\hline $5-8$ & 240 & 19,8 & 625 & 22,1 & 3 & 0,3 & - & - \\
\hline $9-12$ & 275 & 22,7 & 749 & 26,5 & 20 & 2,1 & - & - \\
\hline $13-15$ & 284 & 23,4 & 682 & 24,1 & 36 & 3,8 & 12 & 7,4 \\
\hline $16-19$ & 344 & 28,4 & 651 & 23,0 & 849 & 90,6 & 151 & 92,6 \\
\hline \multicolumn{9}{|l|}{ Sexo } \\
\hline Masculino & 869 & 71,7 & 1591 & 56,3 & 491 & 52,4 & 154 & 94,5 \\
\hline Feminino & 343 & 28,3 & 1237 & 43,7 & 446 & 47,6 & 9 & 5,5 \\
\hline \multicolumn{9}{|l|}{ Raça/Cor da pele } \\
\hline Branca & 102 & 8,4 & 3 & 0,1 & 19 & 2,0 & - & - \\
\hline Preta & 269 & 22,2 & - & - & 62 & 6,6 & - & - \\
\hline Parda & 802 & 66,2 & - & - & 305 & 32,6 & - & - \\
\hline Amarela & 12 & 1,0 & - & - & - & - & - & - \\
\hline Não Informado & 27 & 2,2 & 2825 & 99,9 & 551 & 58,8 & 163 & 100 \\
\hline \multicolumn{9}{|c|}{ Modalidade de atendimento } \\
\hline Intensivo & 141 & 11,6 & 240 & 8,5 & 170 & 18,1 & 27 & 16,6 \\
\hline Semi-intensivo & 524 & 43,2 & 1297 & 45,9 & 401 & 42,8 & 112 & 68,7 \\
\hline Não Intensivo & 547 & 45,1 & 1291 & 45,7 & 360 & 38,4 & 24 & 14,7 \\
\hline \multicolumn{9}{|l|}{ Tipo de atendimento } \\
\hline Inicial & 407 & 33,6 & 1051 & 37,2 & 361 & 38,5 & 51 & 31,3 \\
\hline Continuação & 805 & 66,4 & 1777 & 62,8 & 576 & 61,5 & 112 & 68,7 \\
\hline Total & 1.212 & 100 & 2.828 & 100 & 937 & 100 & 163 & 100 \\
\hline \multicolumn{9}{|c|}{ Fonte: Elaboração própria. } \\
\hline $\begin{array}{r}\text { Nota: Sinal conv } \\
\text { - Dado nu }\end{array}$ & o: & e de arr & damento & & & & & \\
\hline
\end{tabular}

\section{PERFIL NOSOLÓGICO DOS ATENDIMENTOS EM CAPSI}

Observou-se que 73,5\% dos atendimentos nos CAPSis concentraram-se em três grupos da CID-10, a saber: 27,3\% para Transtornos do comportamento e Transtornos emocionais (F90- F98), 26,8\% para algum tipo de retardo mental (F70-F79) e 19,5\% para Transtornos do desenvolvimento psicológico (F80-F89) (Tabela 2). No primeiro grupo, o diagnóstico mais frequente foi transtorno hipercinético; no segundo, retardo mental leve; e no terceiro, transtorno global do desenvolvimento. Os transtornos de desenvolvimento e comportamentais foram 
Revista Baiana de Saúde Pública registrados principalmente na infância, enquanto diagnósticos por transtornos esquizotípicos tenderam a aumentar com a idade, expressando-se com mais evidência entre 16 a 19 anos. O retardo mental foi mais frequentemente diagnosticado entre indivíduos de 9 a 15 anos.

Tabela 2 - Frequência e percentual dos registros de atendimentos nos Centros de Atenção Psicossocial da Infância e Adolescência, por grupo diagnóstico* e faixa etária. Salvador, Bahia, Brasil - 2010-2012

\begin{tabular}{|c|c|c|c|c|c|c|c|c|c|c|c|c|c|}
\hline \multirow{3}{*}{$\begin{array}{l}\text { Código da } \\
\text { CID-10 }\end{array}$} & \multirow{3}{*}{ Diagnóstico } & \multicolumn{12}{|c|}{ Faixa Etária (anos) } \\
\hline & & \multicolumn{2}{|c|}{$1-4$} & \multicolumn{2}{|c|}{$5-8$} & \multicolumn{2}{|c|}{$9-12$} & \multicolumn{2}{|c|}{$13-15$} & \multicolumn{2}{|c|}{$16-19$} & \multicolumn{2}{|c|}{ Total } \\
\hline & & $n$ & $\%$ & $n$ & $\%$ & $\mathbf{n}$ & $\%$ & $n$ & $\%$ & $n$ & $\%$ & \begin{tabular}{l|l}
$n$ \\
\end{tabular} & $\%$ \\
\hline F10-F19 & $\begin{array}{l}\text { Transtornos mentais } \\
\text { e comportamentais } \\
\text { pelo uso de } \\
\text { substância psicoativa }\end{array}$ & - & - & - & - & - & - & 3 & $(100)$ & - & - & 3 & 0,1 \\
\hline F20-F29 & $\begin{array}{l}\text { Esquizofrenia, } \\
\text { transtornos } \\
\text { esquizotípicos e } \\
\text { delirantes }\end{array}$ & - & - & 9 & 1,8 & 42 & 8,2 & 132 & 25,9 & 327 & 64,1 & 510 & 12,6 \\
\hline F30-F39 & $\begin{array}{l}\text { Transtornos do } \\
\text { humor (afetivos) }\end{array}$ & 1 & 0,3 & 30 & 7,8 & 56 & 14,7 & 145 & 37,9 & 150 & 39,3 & 382 & 9,5 \\
\hline F40-F48 & $\begin{array}{l}\text { Transtornos } \\
\text { neuróticos, } \\
\text { transtornos } \\
\text { relacionados ao } \\
\text { stress e transtornos } \\
\text { somatoformes }\end{array}$ & - & - & 22 & 13,0 & 12 & 7,1 & 37 & 21,9 & 98 & 58,0 & 169 & 4,2 \\
\hline F70-F79 & Retardo mental & 29 & 2,7 & 150 & 13,9 & 322 & 29,7 & 307 & 28,3 & 275 & 25,4 & 1083 & 26,8 \\
\hline F80-F89 & $\begin{array}{l}\text { Transtornos do } \\
\text { desenvolvimento } \\
\text { psicológico }\end{array}$ & 117 & 14,9 & 314 & 40,0 & 191 & 24,3 & 100 & 12,7 & 64 & 8,1 & 786 & 19,5 \\
\hline F90-F98 & $\begin{array}{l}\text { Transtornos do } \\
\text { comportamento } \\
\text { e emocionais } \\
\text { que aparecem } \\
\text { habitualmente } \\
\text { na infância ou } \\
\text { adolescência }\end{array}$ & 43 & 3,9 & 340 & 30,1 & 398 & 36,0 & 242 & 21,9 & 81 & 7,3 & 1104 & 27,3 \\
\hline F99 & $\begin{array}{l}\text { Transtorno mental } \\
\text { não especificado }\end{array}$ & - & - & - & - & 3 & 100 & - & - & - & - & 3 & 0,1 \\
\hline
\end{tabular}

PERFIL NOSOLÓGICO DO ATENDIMENTO INFANTOJUVENIL EM CAPS DE ADULTOS

Nos onze CAPS de adultos onde foram observados atendimentos da população infantojuvenil, encontrou-se um total de 96.057 registros, sendo 1,0\% relativo à população 
menor de 19 anos. Encontrou-se nesse subconjunto de usuários, registros de atendimento em todas as faixas etárias, principalmente no grupo entre 16 e 19 anos, representando 90,6\% dos atendimentos realizados em menores de 19 anos. Os diagnósticos mais frequentes nesse grupo corresponderam a esquizofrenias, com 48,3\%; transtornos de humor, com 18,7\%; e transtornos neuróticos relacionados como o estresse, com 12,7\% (Tabela 3).

Tabela 3 - Frequências e percentual dos registros de atendimentos nos Centros de Atenção Psicossocial de Adultos segundo grupo diagnóstico* e faixa etária.

Salvador, Bahia, Brasil -2010-2012

\begin{tabular}{|c|c|c|c|c|c|c|c|c|c|c|c|c|c|}
\hline \multirow{3}{*}{$\begin{array}{l}\text { Código da } \\
\text { CID-10 }\end{array}$} & \multirow{3}{*}{ Diagnóstico } & \multicolumn{10}{|c|}{ Faixa etária (anos) } & \multirow{2}{*}{\multicolumn{2}{|c|}{ Total }} \\
\hline & & \multicolumn{2}{|c|}{$1-4$} & \multicolumn{2}{|c|}{$5-8$} & \multicolumn{2}{|c|}{ 9- 12} & \multicolumn{2}{|c|}{$13-15$} & \multicolumn{2}{|c|}{$16-19$} & & \\
\hline & & $\mathbf{n}$ & $\%$ & $\mathbf{n}$ & $\%$ & $\mathbf{n}$ & $\%$ & $\mathbf{n}$ & $\%$ & $\mathbf{n}$ & $\%$ & $\mathbf{n}$ & $\%$ \\
\hline F00-F09 & $\begin{array}{l}\text { Transtornos mentais } \\
\text { orgânicos, inclusive } \\
\text { sintomáticos }\end{array}$ & - & - & - & - & - & - & - & - & 43 & 100 & 43 & 4,6 \\
\hline F10-F19 & $\begin{array}{l}\text { Transtornos mentais } \\
\text { e comportamentais } \\
\text { pelo uso de } \\
\text { substância psicoativa }\end{array}$ & - & - & - & - & - & - & 14 & 100 & - & - & 14 & 1,5 \\
\hline F20-F29 & $\begin{array}{l}\text { Esquizofrenia, } \\
\text { transtornos } \\
\text { esquizotípicos e } \\
\text { delirantes }\end{array}$ & 3 & 0,7 & - & - & - & - & 4 & 0,9 & 446 & 98,5 & 453 & 48,3 \\
\hline F30-F39 & $\begin{array}{l}\text { Transtornos do } \\
\text { humor (afetivos) }\end{array}$ & 4 & 2,3 & 3 & 1,7 & 6 & 3,4 & - & - & 162 & 92,6 & 175 & 18,7 \\
\hline F40-F48 & $\begin{array}{l}\text { Transtornos } \\
\text { neuróticos, } \\
\text { transtornos } \\
\text { relacionados com } \\
\text { stress e transtornos } \\
\text { somatoformes }\end{array}$ & 20 & 16,8 & - & - & 11 & 9,2 & 18 & 15,1 & 70 & 58,8 & 119 & 12,7 \\
\hline F50-F59 & $\begin{array}{l}\text { Síndromes } \\
\text { comportamentais } \\
\text { associadas a } \\
\text { disfunções } \\
\text { fisiológicas e fatores } \\
\text { físicos }\end{array}$ & 2 & 15,4 & - & - & - & - & - & - & 11 & 84,6 & 13 & 1,4 \\
\hline F70-F79 & Retardo mental & - & - & - & - & 3 & 3,5 & - & - & 82 & 96,5 & 85 & 9,1 \\
\hline F80-F89 & $\begin{array}{l}\text { Transtornos do } \\
\text { desenvolvimento } \\
\text { psicológico }\end{array}$ & - & - & - & - & - & - & - & - & 9 & 100 & 9 & 1,0 \\
\hline F90-F98 & $\begin{array}{l}\text { Transtornos } \\
\text { comportamentais } \\
\text { e emocionais } \\
\text { habitualmente } \\
\text { presentes na } \\
\text { infância ou } \\
\text { adolescência }\end{array}$ & - & - & - & - & - & - & - & - & 26 & 100 & 26 & 2,8 \\
\hline
\end{tabular}

Fonte: Elaboração própria.

Nota: Sinal convencional utilizado:

- Dado numérico igual a zero não resultante de arredondamento.

* Diagnóstico segundo CID-10: Classificação Estatística Internacional de Doenças e Problemas Relacionados a Saúde, $10^{\mathrm{a}}$ Revisão. 
Revista Baiana

de Saúde Pública

\section{PERFIL NOSOLÓGICO DOS ATENDIMENTOS INFANTOJUVENIS EM CAPS-AD}

Dentre os 7.151 atendimentos registrados pelo CAPS-AD no período, 2,3\%

(163) destinou-se ao grupo com idade até 19 anos. Pela especialização do serviço, todos os diagnósticos corresponderam ao grupo de Transtornos mentais e comportamentais pelo uso de substâncias psicoativas (F10-F19). Entre esses jovens, 80,4\% dos atendimentos corresponderam aos transtornos mentais e comportamentais devido ao uso de múltiplas substâncias psicoativas: síndrome de dependência. Por outro lado, dependência específica ao uso do álcool correspondeu a 10,4\% e, para transtornos relacionados com dependência de cocaína, observou-se 9,2\% dos registros (Tabela 4).

Tabela 4 - Frequência e percentual dos registros de atendimentos nos Centros de Atenção Psicossocial para Álcool e outras Drogas segundo faixa etária e diagnóstico*. Salvador, Bahia, Brasil - 2010-2012

\begin{tabular}{|c|c|c|c|c|c|c|c|}
\hline \multirow{2}{*}{$\begin{array}{l}\text { Código da } \\
\text { CID-10 }\end{array}$} & \multirow[t]{2}{*}{ Diagnóstico } & \multicolumn{2}{|c|}{ 13-15 } & \multicolumn{2}{|c|}{ 16-19 } & \multicolumn{2}{|c|}{ Total } \\
\hline & & $\mathbf{n}$ & $\%$ & $\mathbf{n}$ & $\%$ & $\mathbf{n}$ & $\%$ \\
\hline F192 & $\begin{array}{l}\text { Transtornos mentais e comportamentais } \\
\text { pelo uso de múltiplas drogas e uso de } \\
\text { outras substâncias psicoativas: síndrome de } \\
\text { dependência }\end{array}$ & 9 & 6,9 & 122 & 93,1 & 131 & 80,4 \\
\hline F102 & $\begin{array}{l}\text { Transtornos mentais e comportamentais } \\
\text { pelo uso de álcool: síndrome de } \\
\text { dependência }\end{array}$ & - & - & 17 & 100 & 17 & 10,4 \\
\hline
\end{tabular}

Fonte: Elaboração própria.

Nota: Sinal convencional utilizado:

- Dado numérico igual a zero não resultante de arredondamento.

* Diagnóstico segundo CID-10: Classificação Estatística Internacional de Doenças e Problemas Relacionados à Saúde, $10^{a}$ Revisão.

\section{COBERTURA DE CAPS EM SALVADOR}

O Instituto Brasileiro de Geografia e Estatística (IBGE) ${ }^{13}$ considerou uma população total de 2.710 .969 pessoas em 2012 para Salvador e a existência de 19 CAPS nesse mesmo ano. Com base nessa informação, calculou-se, conforme o Ministério da Saúde ${ }^{15}$, a Cobertura para esse serviço, sendo encontrado o indicador de 0,72 CAPS por 100.000 habitantes. Embora não exista um indicador específico para cobertura de CAPS infantojuvenil, estimou-se tal cobertura empregando a população menor de 19 anos para 
2012 em Salvador. Obteve-se um indicador da ordem de 0,8 CAPSi para cada 300.000 mil crianças e adolescentes.

\section{DISCUSSÃO}

Os resultados deste estudo demonstraram que os atendimentos em população masculina foram os mais frequentes, achados similares aos de outra pesquisa ${ }^{11}$. Estudo realizado em Salvador com dados de prontuários encontrou que 68\% dos usuários eram de sexo masculino ${ }^{11}$, seguindo a mesma tendência de resultados dos primeiros CAPSi implantados em diferentes regiões do país, cujo grupo masculino correspondeu a $62,8 \%{ }^{16}$.

Em relação ao perfil nosológico, este estudo sugere um perfil diferenciado entre os grupos etários. Enquanto os transtornos do comportamento e do desenvolvimento foram mais frequentes na população infantil, entre os adolescentes predominaram as Esquizofrenias, Retardo Mental e Transtornos de Humor. Este perfil é similar àquele encontrado em estudo anterior que utilizou dados individuais dos primeiros CAPSis, em que os transtornos de desenvolvimento foram mais frequentes em população menor de dez anos ${ }^{16}$.

Sobre transtornos de comportamento e emocionais, estudos brasileiros demonstraram que esses foram também os mais frequentes entre crianças e adolescentes ${ }^{4,15}$. No estudo realizado em Salvador, com dados de prontuários, os transtornos de comportamento representaram 22,6\% ${ }^{11}$, muito próximo dos achados deste estudo. Portanto, diagnóstico oportuno e tratamento adequado para indivíduos nessas faixas etárias poderiam prevenir problemas sociais graves, como delinquência na adolescência. Estudo realizado com adolescentes em situação de problemas com a justiça no Rio de Janeiro indicou prevalências de $77 \%$ para transtornos de conduta entre os adolescentes, e de 54\% para transtornos de déficit de atenção e hiperatividade; observou ainda que 93\% dos acometidos nunca receberam tratamento ${ }^{17}$.

O volume elevado de atendimentos por retardo mental nos CAPSis $(26,8 \%)$ encontrado neste estudo, principalmente retardo mental leve, vai na mesma direção de estudo anterior desenvolvido em Salvador, no qual o retardo mental foi o diagnóstico mais frequente, com $42 \%{ }^{11}$. Difere, porém, dos achados do estudo sobre os primeiros CAPSi, cuja presença desse transtorno correspondeu a $5,7 \%$ apenas $^{15}$. O fato destaca a prioridade da função articuladora dos CAPSis com os demais níveis de atenção à saúde para a população infantojuvenil, principalmente com a atenção básica em Salvador, para que, ao permitir o atendimento de crianças com retardo mental na atenção básica, possa promover maior cobertura de atendimentos a crianças e adolescestes com transtornos mentais graves nos CAPSi. 
Revista Baiana de Saúde Pública
O número elevado de registros sob o diagnóstico de esquizofrenia poderia ser uma observação positiva, desde que se fizesse acompanhar de diminuição das internações por essa causa, posto que usuários com transtornos esquizofrênicos costumavam ocupar cerca de $50 \%$ dos leitos psiquiátricos ${ }^{18}$. Elevada frequência de atendimento por esquizofrenia entre adolescentes coincide com o relato da literatura sobre o início dessa doença, entre 15 e 25 $\operatorname{anos}^{18}$ e, consequente, o uso de serviços ${ }^{16}$. Chama a atenção nos CAPSis o diagnóstico de esquizofrenia em crianças a partir dos 5 anos, dado que esse diagnóstico seria particularmente escasso nessas faixas etárias, o que também pode sugerir algum tipo de erro no momento de registrar o evento no sistema de informação em saúde.

Os transtornos de desenvolvimento representaram um volume elevado de atendimentos, principalmente na primeira infância. Provavelmente, os percentuais seriam ainda maiores se as equipes de saúde estivessem atentas a essas dificuldades e as famílias fossem melhor informadas, dado que tais transtornos podem ser diagnosticados nos primeiros três anos de vida. O desafio para a compreensão e condução desse tipo de transtorno coloca-se para os serviços de saúde, famílias e sociedade, considerando que o tratamento precoce permite melhoria da qualidade de vida dessas crianças.

Apesar do pequeno valor percentual (2,3\%), encontrou-se no CAPS-AD registro de atendimento por transtornos devido ao consumo de sustâncias psicoativas entre adolescentes, diferindo de estudo anterior, no qual esse diagnóstico não foi relatado ${ }^{16}$. Talvez a ausência de serviços especializados para tais transtornos tivesse contribuído para impedir a presença dessa demanda. A oferta de cuidado especializado para tais transtornos deve ser considerada pela rede pública de atenção em saúde, diante da estimativa de que $5 \%$ da população geral seria usuária de substâncias psicoativas, e 0,6\% teria algum tipo de problema relacionado com esse uso ${ }^{19}$.

No CAPS-AD o consumo de múltiplas substâncias psicoativas responde pela maioria dos diagnósticos entre os adolescentes (80\%), sendo similar aos achados de um estudo desenvolvido em Curitiba, onde 78,3\% dos pacientes de um hospital psiquiátrico para dependentes químicos referiam policonsumo ${ }^{20}$. Outro estudo realizado no Paraná encontrou que $38 \%$ dos usuários do CAPS-AD faziam uso de mais de um tipo de substância psicoativa, e entre a população adolescente essa prevalência alcançava $50 \%{ }^{21}$.

Chama a atenção neste estudo o baixo percentual de atendimentos na modalidade intensiva observado, tendo em vista a substituição das internações psiquiátricas pelo acompanhamento nos CAPS, um dos principais objetivos da reforma psiquiátrica. Isto porque uma das prioridades dos CAPSi seria o atendimento diário de crianças e adolescentes 
que demandassem o referido cuidado. O estudo anterior, dos primeiros CAPSis implantados, também relatou que a modalidade intensiva não ultrapassou $10 \%$ do atendimento ${ }^{16}$.

Conforme o indicador oficial de cobertura de CAPS, Salvador teria uma cobertura considerada muito boa, com 0,72 equipamentos por 100.000 habitantes. Contudo, encontraram-se registros de atendimento da população infantojuvenil em CAPS para atendimento da população adulta, o que pode sugerir oferta mínima de serviços especializados para crianças e adolescentes. A lacuna entre necessidade de tratamento dos transtornos mentais infantojuvenis e acesso à prestação de serviços está mundialmente colocada ${ }^{22}$. Salvador poderia chegar a uma cobertura de 0,76 CAPS por 100.000 habitantes se tivesse um CAPSi a mais, atingindo uma cobertura de 1,2 CAPSi por 300.000 indivíduos até 19 anos. A diferença entre a cobertura atual, com dois CAPSi (0,8/300.000 até 19 anos), e uma cobertura com três CAPSi (1,2/300.000 até 19 anos) é de 33\%. Corrigindo esse dado pela população com plano de saúde em Salvador, que foi de 24\% em 2012, esta diferença estaria coberta.

Esses cálculos, porém, baseiam-se no atual indicador de cobertura do Ministério da Saúde e talvez não reflita a real situação do cuidado em saúde mental de crianças e adolescentes. Alguns autores têm chamado a atenção sobre as possíveis falácias desse indicador em relação às regiões ${ }^{23}$. Em outro estudo realizado em São Paulo, foram feitos cálculos de cobertura de CAPSi por total de habitantes e por prevalência de transtornos mentais na infância. Em ambos, o número de CAPSi era insuficiente ${ }^{7}$. O Ministério de Saúde, em 2012, reconheceu as limitações do atual indicador de CAPS e enfatizou a importância de construir novos indicadores sensíveis à cobertura de CAPS infantojuvenil24. Até o momento não há informação sobre novos indicadores.

Diante de um estudo que utilizou dados públicos da APAC/SIA, cabe discutir algumas limitações decorrentes do uso dos Sistemas de Informação em Saúde (SIS). Os dados registrados nesses sistemas dependem, desde a coleta até sua disponibilização e uso, do ponto de vista ou grau de conhecimento e tecnologia de que se dispõe e das definições prévias do que se queira mostrar ${ }^{25}$. Neste estudo em particular não foi possível obter dados de períodos similares entre as diferentes unidades. Observou-se ainda falta de preenchimento para algumas variáveis, consideradas importantes no momento de avaliar a qualidade dos $\operatorname{dados}^{26}$, principalmente a variável raça/cor da pele; atributos como a cobertura, completude, confiabilidade e validade.

A ausência de identificação nominal comprometeria inferências populacionais, já que mais de um atendimento poderia ter ocorrido para a mesma pessoa. 
Revista Baiana de Saúde Pública

\section{CONSIDERAÇÕES FINAIS}

Os resultados enfatizaram a importância de um trabalho articulado com diferentes níveis de atenção em saúde e demais setores vinculados, visando o adequado aproveitamento dos serviços de saúde especializados. Condições como os transtornos de comportamento e emocionais, e também o retardo mental, poderiam ser assistidos com apoio da rede básica e do setor educativo e social no território, e não serem prioritariamente encaminhados aos serviços especializados como os CAPSis, que foram desenhados para atendimentos de transtornos mentais graves.

Conclui-se que o perfil nosológico dos CAPS infantis caracteriza-se pelo predomínio dos transtornos de aprendizagem. O atendimento infantojuvenil em CAPS para adultos sugere déficit do número de CAPSi.

A presença de atendimentos por diagnósticos relacionados ao abuso de substâncias psicoativas entre os adolescentes coloca em evidência a importância de investimento na formação de profissionais para atender essa demanda.

O estudo revela a necessidade de aprimorar o registro de atividades dos Sistemas de Informação em Saúde (SIS), considerando que o uso de dados públicos do Datasus contribui para produzir conhecimentos epidemiológicos relativos aos serviços de saúde mental para a infância e a adolescência. Recomendam-se novas pesquisas que aprofundem o conhecimento da utilização desta nova rede de serviços no cenário da reforma psiquiátrica brasileira.

\section{AGRADECIMENTOS}

À Coordenação de Aperfeiçoamento de Pessoal de Nível Superior (Capes), pela bolsa de Mestrado concedida a Grey Yuliet Ceballos Garcia durante seus estudos de mestrado no Instituto de Saúde Coletiva da Universidade Federal de Bahia no período de 2013 a 2015.

\section{COLABORADORES}

1. Concepção do projeto, análise e interpretação dos dados: Grey Yuliet Ceballos Garcia, Darci Neves dos Santos e Eduardo Luiz Andrade Mota.

2. Redação do artigo e revisão crítica relevante do conteúdo intelectual: Grey Yuliet Ceballos Garcia, Darci Neves dos Santos e Eduardo Luiz Andrade Mota.

3. Revisão e/ou aprovação final da versão a ser publicada: Zenith Rosa Silvino.

4. Ser responsável por todos os aspectos do trabalho na garantia da exatidão e integridade de qualquer parte da obra: Grey Yuliet Ceballos Garcia, Darci Neves dos Santos e Eduardo Luiz Andrade Mota. 


\section{REFERÊNCIAS}

1. Organización Mundial de la Salud. Carga mundial de trastornos mentales y necesidad de que el sector de la salud y el sector social respondan de modo integral y coordinado a escala de país. Documento WHA65.4, 25 de maio de 2012. Ginebra; 2012 [citado 2015 dez 7]. Disponible: http:// www.paho.org/bulletins/index.php?option $=$ com_content\&view $=$ article\& $\mathrm{id}=1165$ :resolucion-wha65.4-sobre-salud-mental-ahora-disponible-eninternet\&ltemid $=0$ \&lang $=\mathrm{es}$

2. World Health Organization. Child and adolescent Atlas: resources for child and adolescent mental health. Geneva; 2005 [cited 2014 Jul 2]. Available from: http://www.who.int/mental_health/resources/Child_ado_atlas.pdf

3. Almeida-Filho N. Estudos de prevalência de desordens mentais na infância em zona urbana de Salvador. Rev Bras Psiquiatr. 1982;31:225-36.

4. Goodman R, Neves-dos-Santos D, Robatto-Nunes AP, Pereira-de-Miranda D, Fleitlich-Bilyk B, Almeida Filho N. The Ilha de Maré study: a survey of child mental health problems in a predominantly African-Brazilian rural community. Soc Psychiatry Psychiatr Epidemiol. 2005 Jan [cited 2015 Nov 18];40(1):11-7. Available from: http://link.springer.com/article/10.1007/s00127-005-0851-z

5. Paula CS, Duarte CS, Bordin IA. Prevalence of mental health problems in children and adolescents from the outskirts of São Paulo City: treatment needs and service capacity evaluation. Rev Bras Psiquiatr [internet]. 2007 [cited 2015 Nov 16];29(1):11-7. Available from: DOI: 10.1590/S151644462006005000012

6. Kieling K, Baker-Henningham H, Belfer ML, Conti G, Ertem I, Omigbodun $\mathrm{O}$, et al. Child and adolescent mental health worldwide: evidence for action. Lancet [internet]. 2011 [cited 2015 Dec 13];378:1515-25. Available from: DOI: 10.1016/S0140-6736(11)60827-1

7. Paula CS, Lauridse-Ribeiro E, Wissow L, Bordin I, Evans-lacko S. How to improve the mental health care of children and adolescents in Brazil: Actions needed in the public sector. Rev Bras Psiquiatr [internet]. 2012 [cited 2015 Nov 28];34:334-41. Available from: DOI: 10.1016/j.rbp.2012.04.001

8. Associação Brasileira de Psiquiatria. Cerca de 5 milhões de crianças demonstram problemas mentais. Rio de Janeiro; 2008 [citado 2014 jul 3]. Disponível em: http://www.abp.org.br/medicos/pesquisas/

9. World Health Organization. Mental Health Atlas 2011. Geneva; 2011[cited 2015 Dec 7]. Available from: http://www.who.int/mental_health/publications/ mental_health_atlas_2011/en 
Revista Baiana de Saúde Pública
10. Brasil. Ministério de Saúde. Saúde mental no SUS: os Centros de Atenção Psicossocial. Brasília; 2004 [citado 2015 nov 18]. Disponível em: http://www. ccs.saude.gov.br/saude_mental/pdf/sm_sus.pdf

11. Santos DN, Carvalho MM, Pinho MA, Nunes APR. Atendimento em psiquiatria da infância e adolescência em serviços públicos de Salvador. Rev baiana saúde pública. 2005;29(1):35-42.

12. World Health Organization. Adolescent health. Geneva; 2017 [cited 2015 Dec 7]. Available from: http://www.who.int/topics/adolescent_health/en/

13. Instituto Brasileiro de Geografia e Estatística. Censo de 2010. Brasília; 2010 [citado 2015 nov 20]. Disponível em: http://cidades.ibge.gov.br/xtras/temas. php?lang $=\&$ codmun $=292740 \&$ idtema $=1 \&$ search $=$ bahia $\mid$ salvador $\mid$ censodemografico-2010:-sinopse-

14. Brasil. Lei n.10.216, de 6 de abril de 2001. Dispõe sobre a proteção e os direitos das pessoas portadoras de transtornos mentais e redireciona o modelo assistencial em saúde mental. Brasília; 2001 [citado 2015 nov 18]. Disponível em: http://www.planalto.gov.br/ccivil_03/leis/LEIS_2001/L10216.htm

15. Brasil. Ministério de Saúde. Relatório de gestão 2003-2006. Saúde mental no SUS: acesso ao tratamento e mudanças do modelo de atenção. Brasília; 2007 [citado 2014 jul 3]. Disponível em: http://bvsms.saude.gov.br/bvs/ publicacoes/07_0416_M.pdf

16. Hoffmann MC, Santos D, Mota EL. Caracterização dos usuários e dos serviços prestados por Centros de Atenção Psicossocial Infanto-Juvenil [internet]. Cad saúde pública. 2008 [citado 2015 dez 18];24(3):633-42. Disponível em:

DOI: 10.1590/S0102-311X2008000300017

17. Andrade RC, Silva VA, Assumpção FB. Preliminary data on the prevalence of psychiatric disorders in Brazilian male and female juvenile delinquents. Braz J Med Biol Res [internet]. 2004 [cited 2015 Dec 20];37:1155-60. Disponível em: DOI: 10.1590/S0100-879X2004000800005

18. Kaplan H, Sadock B, Grebb J. Esquizofrenia. In: Kaplan H, Sadock B, Grebb J. Compendio de Psiquiatria. 7a ed. Porto Alegre: Artes Médicas; 1997. p.104-16.

19. Schmidt MI, Duncan BB, Lopes AA. Epidemiologia clínica: como empregar evidências epidemiológicas na prática clínica. In: Almeida-Filho N, Barreto M, coordenadores. Epidemiologia \& saúde: fundamentos, métodos aplicações. Rio de Janeiro: Guanabara Koogan; 2012. p. 350-62.

20. Capistrano F, Ferreira A, Silva T, Kalinke L, Maftum M. Perfil sociodemográfico e clínico de dependentes químicos em tratamento: análise de prontuários. Esc 
Anna Nery [internet]. 2013 [citado 2015 nov 28];17(2):234-41. Disponível em: DOI: 10.1590/S1414-81452013000200005

21. Macagnan J, Menetrier J, Schiavoni D. Perfil dos usuários de um Centro de Atenção Psicossocial no município de Francisco Beltrão - Paraná. Biosaúde. 2014;16(2);34-44.

22. Organización Mundial de la Salud. Carga mundial de trastornos mentales y necesidad de que el sector de la salud y el sector social respondan de modo integral y coordinado a escala de país. Documento EB130/9 1 de dezembro de 2011. Ginebra; 2011 [citado 2014 jul 2]. Disponible: http://apps.who.int/ gb/ebwha/pdf_files/EB130/B130_9-sp.pdf

23. Goncalves VM, Abreu PS, Candiago RH, Saraiva SS, Lobato MI. A falácia da adequação da cobertura dos Centros de Atenção Psicossocial no estado do Rio Grande do Sul. Rev Psiquiatr Rio Gd Sul [internet]. 2010 [citado 2014 ago 28];32(1):16-8. Disponível em: DOI: 10.1590/S0101-81082010000100003

24. Brasil. Ministério de Saúde. Secretaria de Atenção à Saúde. Departamento de Ações Programáticas Estratégicas. Coordenação Geral de Saúde Mental, Álcool e Outras Drogas. Saúde Mental em Dados - 11, Ano VII, no 11. Informativo eletrônico. Brasília 2012 out [citado 2014 ago 28]. Disponível em: http://psiquiatriabh.com.br/wp/wp-content/uploads/2015/01/Dados-darede-assistencial-brasileira-2012-Ministerio-da-Saude.pdf

25. Moraes IHS. Sistemas de Informação em Saúde: patrimônio da sociedade brasileira. In: Paim JS, Almeida Filho N, organizadores. Saúde Coletiva: teoria e prática. Rio de Janeiro: MedBook; 2014. p. 649-65.

26. Mota E, Alazraqui M. Informação em Saúde Coletiva. In: Paim JS, Almeida Filho N, organizadores. Saúde Coletiva: teoria e prática. Rio de Janeiro: MedBook; 2014. p. 195-9.

Recebido: 12.4.2015. Aprovado: 11.3.2016. Publicado: 8.11.2017. 